\title{
La violencia obstétrica en la vulneración de los derechos humanos de las mujeres
}

\author{
Obstetric violence in the violation of women's human rights \\ María Daniela Ramírez Saucedo,* César Hernández Mier,łGrey Yuliet Ceballos García§
}

\begin{abstract}
RESUMEN
Introducción: La violencia obstétrica (VO) es una forma de violencia de género que se ha convertido en una problemática mundial, vulnerando los derechos humanos (DD. HH.) de las mujeres, en particular aquellos sexuales y reproductivos, repercutiendo negativamente en su autonomía, su sanidad física, sicoemocional e incluso social. El abordaje de esta rudeza en la obstetricia, a partir del análisis crítico feminista, refleja que sus bases radican en prejuicios y estereotipos machistas que conciben la reproducción como un acto inherente a la naturaleza femenina. De esta forma, el abuso obstétrico en los establecimientos sanitarios no respeta, no protege, ni garantiza los DD. HH. de las mujeres. Objetivo: Delimitar los DD. HH. quebrantados a través de la VO, al igual que su impacto en la salud y dignidad de la mujer. Conclusiones: Aunque la definición y caracterización de la VO es reciente, es importante abordarla de manera prioritaria. Es imprescindible incrementar la perspectiva de género en las instituciones de salubridad, de manera que se salvaguarde el honor de las féminas, eliminando los prejuicios y estereotipos existentes en torno a la reproducción. Ingresar a una institución sanitaria no implica que las mujeres pierdan sus derechos, su calidad de humanas, ni sus facultades de toma de decisiones, libertad y dignidad. Es necesario incrementar la investigación en el campo de la VO a fin de obtener una mayor visión interpretativa sobre este fenómeno, sus causas y consecuencias, y con ello lograr su erradicación paulatina.
\end{abstract}

Palabras clave: Violencia obstétrica, violencia de género, derechos sexuales y reproductivos, derechos humanos de las mujeres.

\begin{abstract}
Introduction: Obstetric violence is a form of gender violence, it has become a global problem, violating the human rights of women, in particular their sexual and reproductive rights, negatively affecting their autonomy, their physical, psycho-emotional, and even social health. The approach to obstetric violence based on feminist critical analysis reflects that its bases lie in prejudices and sexist stereotypes that conceive reproduction as an act inherent to female nature. Thus, obstetric violence in health institutions does not respect, protect, or guarantee the human rights of women. Objective: To define the human rights violated through obstetric violence, as well as its impact on the health and dignity of women. Conclusions: Although the definition and characterization of obstetric violence is recent, it is important to address it as a priority. It is essential to increase the gender perspective in health institutions, to safeguard the dignity of women, eliminating existing prejudices and stereotypes regarding reproduction. Entering a health institution does not imply that women lose their rights, their human faculties, or their decision-making powers, freedom and dignity. It is necessary to increase research in the field of obstetric violence in order to obtain a greater interpretive vision of this phenomenon, its causes and consequences, and thereby achieve its gradual eradication.
\end{abstract}

Keywords: Obstetric violence, gender violence, sexual and reproductive rights, human rights of women.
* Licenciada en Enfermería, estudiante de Maestría en Derechos Humanos. Universidad Autónoma de San Luis Potosí, México.

₹ Máster en Medicina Forense, profesor investigador de la Universidad Autónoma de San Luis Potosí, México.

${ }^{\S}$ Máster en Salud Comunitaria, profesora investigadora de la Universidad de Antioquia, Colombia.

Correspondencia: MDRS, mdaniela_1406@hotmail. com

Conflicto de intereses: LOS autores declaran no tener conflicto de intereses. Este artículo es resultado del trabajo de grado de maestría de María Daniela Ramírez Saucedo. Citar como: Ramírez SMD, Hernández MC, Ceballos GCY. La violencia obstétrica en la vulneración de los derechos humanos de las mujeres. Rev CONAMED. 2021; 26(3): 149-155. https:// dx.doi.org/10.35366/101680 Financiamiento: Este artículo hace parte del trabajo de grado de maestría de María Daniela Ramírez Saucedo. No se recibió financiación externa.

Recibido: 05/04/2021 Aceptado: 27/07/2021. 


\section{INTRODUCCIÓN}

El acceso a una vida libre de violencia es un derecho humano básico interdependiente a los derechos de dignidad, desarrollo insubordinado de la personalidad y a la salud. Garantizar estas potestades a las mujeres requiere erradicar toda práctica de agresión física, sicoemocional, sexual, simbólica e institucional contra ellas, incluyendo la VO, la cual es definida como cualquier conducta, acción u omisión, realizada por personal de sanidad (PS) que de manera directa o indirecta, en el ámbito público o privado, afecte el cuerpo y los procesos reproductivos de las féminas, expresada en un trato deshumanizado, abuso de la medicalización y patologización de los procesos naturales.' La VO se manifiesta en toda acción violenta que pueda aquejar a las mujeres durante las fases reproductivas (embarazo, parto y puerperio), es en especial durante el alumbramiento cuando las féminas se vuelven más vulnerables de sufrirla.

Investigaciones en torno a la calidad de la atención obstétrica identifican un trato irrespetuoso y ofensivo durante el nacimiento en instituciones sanitarias (IS), donde se transgreden los DD. HH. ${ }^{2}$ Según datos de la Organización Mundial de la Salud (OMS), la VO se presenta aproximadamente en una de cada 3.5 atenciones obstétricas, ${ }^{3}$ volviéndola un problema de salud pública y de DD. HH., cuya erradicación es prioritaria. En México, el Instituto Nacional de Estadística, Geografía e Informática (INEGI) fue la primera instancia en reportar hallazgos acerca de VO, a través de los resultados de la Encuesta Nacional sobre la Dinámica de las Relaciones en los Hogares (ENDIREH, 2016), mostrando que de 2.9 millones de mujeres (15-49 años de edad) que fueron atendidas por parto o cesárea, 33.4\% sufrieron algún tipo de maltrato. ${ }^{4}$

En la República Mexicana, el machismo continúa latente en diversos aspectos sociales, incluyendo la atención médica. La cultura patriarcal está enraizada en la cultura, tanto que algunas mujeres pueden llegar a normalizar la violencia de género (VG) suscitada en el servicio de obstetricia. La institucionalización del nacimiento de bajo riesgo ha contribuido a patologizarlo, viendo la intervención hospitalaria como una necesidad, en el cual pueden ocurrir actos de abuso de autoridad, arrebato del protagonismo de la mujer en el parto, y vulneraciones de sus DD. HH.

El presente artículo, con un diseño exploratorio, mediante el empleo de hermenéutica jurídica y revisión de la bibliografía existente, tiene el objetivo de delimitar los DD. HH. infringidos a través de la $V O$, al igual que su impacto en la salud y respetabilidad de las mujeres que la sufren.

\section{LA VIOLENCIA OBSTÉTRICA COMO FORMA DE VIOLENCIA DE GÉNERO}

La VO es una forma de VG y no debe confundirse con la negligencia médica, aunque tanto la VO como dicha negligencia involucran errores durante el proceso de atención; a pesar de contar con los conocimientos necesarios para atender una condición de salud, la VO tiene su origen en cuestiones de género, es decir, es provocada por la asimetría en las relaciones de poder entre mujeres y hombres, donde se perpetúa la subordinación y desvalorización de lo femenino frente a lo masculino, producto de una cultura patriarcal. La Organización de las Naciones Unidas (ONU) declara que la VO está tan normalizada, que en muchos países todavía no se considera VG, es decir, es una agresión invisible, y no reconocida socialmente, oculta tras protocolos hospitalarios, ${ }^{5}$ siendo a su vez una forma de violencia simbólica e institucional, buscando generar aceptación o naturalización de la misma por parte de sus víctimas.

Existe consenso entre teóricas feministas al considerar que la relación entre el PSy las mujeres durante la diligencia obstétrica es desigual y basada en relaciones de poder. Las actitudes, roles y normas conductuales, políticas e institucionales, codifican la práctica de la medicina en una serie de estereotipos o prejuicios en los que se concibe a la reproducción como un acto inherente a la naturaleza femenina, donde toda consecuencia (positiva o negativa) ha de ser aceptada obediente y pasivamente por parte de la madre en ciernes.

\section{LA VIOLENCIA OBSTÉTRICA Y SU REGULACIÓN}

La práctica médica se encuentra regulada en todo el mundo, por tratados globales, leyes nacionales y locales. En tal caso, la VO no es la excepción. A 
nivel internacional se ha alertado acerca de la importancia del respeto de los derechos sexuales y reproductivos (DSR), considerando la salud sexual y reproductiva como una parte inalienable, integral e indivisible de los DD. $\mathrm{HH}$. universales, ${ }^{6}$ tal es el caso de la OMS, con la Convención Sobre la Eliminación de Todas las Formas de Discriminación Contra la Mujer (CEDAW, 1979), la Declaración de Naciones Unidas sobre la Eliminación de la Violencia contra la Mujer (1993), la Cuarta Conferencia Mundial sobre la Mujer (1995), la Convención Interamericana para Prevenir, Sancionar y Erradicar la Violencia contra las Mujeres «Convención Belem De Pará» (1996) y el Consenso de Montevideo sobre población y desarrollo (2013).

En Latinoamérica, los DRS son reconocidos por la Institución Interamericana de Derechos Humanos (IIDH) desde el 2008,7 tienen valía internacional y son jurídicamente vinculantes. La IIDH los define como el conjunto de DD. HH. que tienen que ver con la salud reproductiva y más ampliamente con todos aquellos que inciden en la reproducción humana; a esta definición se debería sumar la esfera de sexualidad humana, puesto que los DD. HH. no se limitan solamente a la función reproductiva. Venezuela, Argentina y México reconocen la VO en su leyes de acceso a las mujeres a una existencia exenta de violencia, y las han adaptado buscando su regulación o tipificación, siguiendo las recomendaciones de las convenciones internacionales.

La VO se encuentra incorporada en la ley estatal de sólo 17 entidades de la República Mexicana, además esto contrasta con la Ley General de Acceso de Las Mujeres a una Vida Libre de Violencia (LGAMVLV) donde aún no se incluye, a pesar de múltiples iniciativas encaminadas a su incorporación (Tabla 7).

\section{LOS DERECHOS HUMANOS VULNERADOS EN LA VIOLENCIA OBSTÉTRICA}

En este país, la Comisión Nacional de Derechos Humanos (CNDH) reconoce y conceptualiza la VO como una modalidad de violencia institucional y de género, cometida por prestadores de servicios sanitarios por una deshumanizada atención durante el proceso reproductivo, generándose afectación física, psicológica o moral, o incluso la pérdida de la vida de la madre, del feto o del recién nacido, derivado de la prestación de servicios médicos, abuso de medicalización y patologización de procedimientos naturales. ${ }^{8}$ El hecho de definirla contribuye a reconocer sus causas, manifestaciones y consecuencias, del mismo modo que las medidas pertinentes con tal de erradicarla.

Lo anterior permite identificar que la VO puede vulnerar los distintos DD. HH. que poseen las mujeres, entre ellos el derecho a la vida. Este derecho implica que toda persona disfrute de su ciclo existencial sin interrupciones de agentes externos, ya que el Estado debe respetar la existencia humana, en el deber negativo de evitar su privación, y en el deber positivo de proteger y garantizar la vida de quienes se encuentren bajo su jurisdicción. ${ }^{8}$ Dicho derecho se transgrede a través de acciones $u$ omisiones que tienen por consecuencia la pérdida de la vida de la mujer, tales como desestimar los padecimientos manifestados por ella, descuidar la vigilancia estrecha de la diada materno-fetal, abandonar a la paciente, medicalizar y patologizar los procesos naturales.

Al mismo tiempo, se identifica la vulneración del derecho a la integridad personal, que es considerada en múltiples tratados ${ }^{9}$ e incluye las dimensiones física, psicológica y moral; afectaciones a este derecho generan inestabilidad en cualquiera de sus aristas, por lo que la VO incide directa e indirectamente en la integridad de las féminas. Ejemplos de este quebranto se identifican en la falta de trato oportuno a las mujeres y en la ejecución de algunas maniobras que incrementan el riesgo de lesiones en el canal vaginal y en el feto, que a su vez están prohibidas en la NOM-007-SSA2-2016.10 En el daño a la dimensión psicológica y moral, se identifican prácticas de infantilización, no resolver cuestionamientos o hacerlas sentir culpables por cualquier resultado negativo durante el parto.

Se infringe el derecho a la igualdad y no discriminación," lo cual representa, como lo expresan la OMS y la CNDH, una preocupación particular por las féminas que viven en situación de vulnerabilidad (afrodescendientes, migrantes, mujeres que viven con $\mathrm{VIH}$, indígenas, con discapacidad, menores de edad, entre otras), generándose una doble transgresión, en primer lugar, por cuestiones de género y en segundo por la condición social de cada mujer. 


\begin{tabular}{|c|c|c|c|c|c|}
\hline Entidad & $\begin{array}{l}\text { Considera } \\
\text { violencia } \\
\text { obstétrica }\end{array}$ & Definición & $\begin{array}{l}\text { Componente de } \\
\text { derechos sexuales } \\
\text { y reproductivos }\end{array}$ & $\begin{array}{l}\text { Componente de } \\
\text { dignidad }\end{array}$ & $\begin{array}{l}\text { Componente de } \\
\text { patologización y } \\
\text { medicalización }\end{array}$ \\
\hline Ley Federal & No & & & & \\
\hline Aguascalientes & Sí & Sí & No & Sí & Sí \\
\hline Baja California & No & & & & \\
\hline Baja California Sur & Sí & Sí & No & Sí & Sí \\
\hline Campeche & No & & & & \\
\hline Chiapas & Sí & Sí & No & Sí & Sí \\
\hline Chihuahua & No & & & & \\
\hline CDMX & No & & & & \\
\hline Colima & Sí & Sí & Si & Sí & Sí \\
\hline Durango & Sí & Sí & No & Sí & Sí \\
\hline Estado de México & Sí & Sí & No & Sí & Sí \\
\hline Guanajuato & Sí & Sí & No & Sí & Sí \\
\hline Guerrero & No & & & & \\
\hline Hidalgo & No & & & & \\
\hline Jalisco & No & & & & \\
\hline Michoacán & No & & & & \\
\hline Morelos & Sí & Sí & No & Si & Sí \\
\hline Nayarit & No & & & & \\
\hline Nuevo León & No & & & & \\
\hline Oaxaca & No & & & & \\
\hline Puebla & Sí & Sí & No & Sí & Sí \\
\hline Querétaro & Sí & Sí & No & Sí & Sí \\
\hline Quintana Roo & Sí & Sí & No & Sí & Sí \\
\hline San Luis Potosí & Sí & Sí & No & Sí & Sí \\
\hline Sinaloa & No & & & & \\
\hline Sonora & No & & & & \\
\hline Tabasco & No & & & & \\
\hline Tamaulipas & Sí & Sí & No & Sí & Sí \\
\hline Tlaxcala & Sí & Sí & No & Sí & Sí \\
\hline Veracruz & Sí & Sí & No & Sí & Sí \\
\hline Yucatán & Sí & No & Sí & Sí & No \\
\hline Zacatecas & Sí & Sí & No & Sí & Sí \\
\hline
\end{tabular}

Fuente: Elaboración propia a partir del análisis de la Ley General de Acceso a las Mujeres a una Vida Libre de Violencia a nivel federal y estatal. 
Se viola el derecho a la libertad y la autonomía reproductiva, la cual representa la libertad de decidir de manera autónoma, responsable e informada el número y espaciamiento de los hijos. Tal derecho es interdependiente al desarrollo soberano de la personalidad. La CEDAW reconoce la importancia del acceso de las mujeres a la información, educación y medios que les permitan ejercer este derecho.12 Esta vulneración se ejemplifica en prácticas de esterilización forzada, la imposición de método anticonceptivo, procedimientos procurando acelerar el trabajo de parto, negación del bloqueo epidural (si la parturienta lo solicita), burlas, descalificaciones e insultos ante sus decisiones reproductivas.

Se priva del derecho a la información y al libre consentimiento informado, los cuales abordan la libertad de las féminas a solicitar, recibir y difundir información, al igual que autorizar procedimientos o tratamientos relacionados con su salud. La información que reciben las mujeres tiene que ser veraz, comprensible, objetiva, imparcial, sin prejuicios e inmune a cualquier coerción. Su violación se observa al subestimar la opinión de la mujer en torno a su situación de sanidad con lo que se anula su capacidad de decisión, solicitar el consentimiento respecto a algún procedimiento al acompañante de la ella (frecuentemente el esposo o pareja) y no directamente a ella, lo que le impone un rol o estatus de incapacidad en la toma de decisiones, no prestar importancia a que reciba información de riesgos y beneficios, ejecutando discriminación, amenazas o presiones, asimismo realizar procedimientos invasivos rutinizados, dígase la episiotomía y tactos vaginales sin consentimiento.

Se vulnera el derecho a la protección a la salud, el cual tiene fundamento en el artículo $4^{\circ}$ constitucional en México, ${ }^{13}$ cuyo cumplimiento consiste en que cada persona alcance el nivel más alto posible de sanidad física y mental, del mismo modo que el disfrute de las facilidades, bienes y servicios para alcanzarlo. Esto involucra que las mujeres reciban atención oportuna, de calidad, profesional, éticamente responsable, respetuosa y digna. Acciones que transgreden este derecho incluyen la ejecución de prácticas médicas sin evidencia científica, reconocidas así por la International Mother-Baby Childbirth Initiative (IMBCI) ${ }^{14}$ por ejemplo: tricotomía púbica, enema evacuante, exámenes vaginales repetitivos, restricción de ingesta de alimentos y líquidos, confinamiento dentro de cama, instalación de líquidos intravenosos sin necesidad clínica, posición de litotomía exclusiva durante la fase expulsiva del parto, episiotomía, uso de fórceps; así mismo el sustituir las actividades asistenciales del personal de contrato con estudiantes de áreas de la salud, ${ }^{15}$ infraestructura y equipamiento inadecuados, personal obstétrico insuficiente, y el no aplicar la lex artis ad hoc.

Las acciones descritas que infringen los otros derechos también transgreden el derecho a acceder a una vida libre de violencia, el cual fue planteado inicialmente en la Convención Belém do Pará y posteriormente fue ratificado y promulgado como Ley General en México (2007). Representa el derecho de toda mujer a que ninguna acción u omisión basada en el género, le cause daño o sufrimiento físico, psicológico, patrimonial, económico, sexual o moral, involucrando todos los ámbitos de su existencia. ${ }^{16}$ Ese derecho puede quebrantarse a través de la relación jerárquica de poder entre el PS y la mujer, con base en una diferencia de funciones, habilidades y conocimientos, y al predominio de una cultura sexista y discriminatoria de género, lo cual conlleva al maltrato de las féminas; a su vez, engloba características de todas las libertades anteriormente analizadas. Su vulneración se identifica en actos de hostigamiento sexual, trato deshumanizado, burlas, amenazas, insultos, descalificaciones, gritos, dificultad de las mujeres al intentar manifestar dudas, miedos o inquietudes, igual que el impedimento para la expresión de emociones por mala actitud del PS, negación de tratamiento (sin justificación clínica), exámenes o procedimientos dolorosos, igual que cualquier tipo de agresión física o sicológica.

\section{CONCLUSIÓN}

En esta república se continúa desestimando la importancia de la mujer como sujeta de derechos en diversos ámbitos, incluido el de atención a la salud, lo cual debe corregirse con el fin de que las féminas alcancen un nivel de bienestar más allá de la dimensión física, es decir, que se extienda hasta el plano sicoemocional, social y relativo a su honorabilidad humana. Ingresar a una institución de salubridad no implica que las mujeres pierdan sus 
derechos, su facultad de humanas, ni sus prerrogativas de toma de decisiones, libertad y dignidad.

Aunque la definición y caracterización de la VO es reciente, es importante abordarla de manera prioritaria al ser parte de la mejora continua de la práctica médica. Una herramienta imprescindible que procura lograrlo es incrementar la perspectiva de género en los institutos de salud, de manera que se salvaguarde el honor de las mujeres y se eliminen los prejuicios y estereotipos existentes en torno a la reproducción.

Un avance de dicha perspectiva en las instituciones sanitarias es la creación de la Iniciativa del Parto Humanizado, a modo de respuesta frente al modelo tradicional de servicio médico que desestima el nacimiento como proceso natural, que no toma en cuenta sus necesidades emocionales, culturales y sociales, ni las recomendaciones sustentadas en el enfoque basado en evidencias y principios propuestos por la OMS..$^{17}$ También se ha acuñado el término de «nacimiento humanizado» el cual incluye todo el proceso: desde el embarazo hasta el puerperio tardío, e involucra la atención a la persona recién nacida y su desarrollo futuro, en aras de la disminución de actos de violencia y bajo el respeto de los DD. $\mathrm{HH}$.

La ejecución de la Iniciativa del Parto Humanizado en las organizaciones sanitarias ha de involucrar una visión centrada en la señora como protagonista de su alumbramiento, donde las mujeres y los miembros de la familia puedan elegir la forma en la que quieren que sus hijos nazcan, enmarcado en un cuidado profesional capacitado no sólo en el ámbito clínico y práctico sino también en la calidad y la dignificación de la atención.

La inclusión de la perspectiva de género desde la formación universitaria en el campo de la obstetricia puede contribuir de forma importante en la erradicación de la VO. Esto conlleva a que los organismos educativos formen a los estudiantes en un marco de respeto hacia los DD. HH. de las mujeres (integrando a la vez la normativa internacional y nacional existente), y el enfoque de medicina basada en evidencia, que en el ámbito obstétrico busca eliminar la ejecución de prácticas rutinarias que, al no demostrar sustento científico, se convierten en formas de $V O$.

La perspectiva de los DD. HH. debe fomentarse tanto en el ámbito formativo de las univer- sidades como en los profesionales activos en las instituciones de salud, con el fin de que, en primer lugar, el personal reconozca todos los DD. HH. que poseen las mujeres que atienden, en aras de centrar la práctica clínica en el mantenimiento de su bienestar y en la garantía al acceso a una vida exenta de agresiones, es decir, el personal sanitario tiene que convertirse en un verdadero agente de cambio. Así mismo es necesario incrementar la investigación en el campo de la VO para lograr obtener una visión más interpretativa sobre sus causas y consecuencias y medidas a fin de lograr su erradicación paulatina.

\section{AGRADECIMIENTOS}

Agradecemos a todas las mujeres que en nuestra vida personal y profesional nos inspiraron para realizar investigación en este campo.

\section{REFERENCIAS}

1. Medina G. Violencia Obstétrica. RDFyP. 2009; 4 (1): 16-21.

2. OMS. Human Reproduction Programme [Internet]. Ginebra: OMS; 2014 [consultado 12 Dic 2020]. Disponible en: https://www.who.int/reproductivehealth/topics/maternal_ perinatal/statementchildbirth/es/

3. OMS. Informe de la OMS destaca que la violencia contra la mujer es un problema de salud global de proporciones epidémicas. [Internet]. Ginebra: OMS; 2013 [consultado 12 Dic 2020]. Disponible en: https://www.who. int/mediacentre/news/releases/2013/violence_against_ women_20130620/es/

4. INEGI. Encuesta Nacional sobre la Dinámica de las Relaciones en los Hogares [Internet]. México: INEGI; 2017 [Consultado 22 Jun 2021]. Disponible en: https://www. inegi.org.mx/contenidos/programas/endireh/2016/doc/ endireh2016_presentacion_ejecutiva.pdf

5. Organización de las Naciones Unidas (ONU). Informe de la Cuarta Conferencia Mundial sobre la Mujer [Internet]. Sep 2015 [consultado 20 Dic 2020]. Disponible en: http://www. un.org/womenwatch/daw/beijing/pdf/Beijing\%20full\%20 report\%20s.pdf

6. Belli LF. La violencia obstétrica: otra forma de violación a los derechos humanos. Rev Redbioét/UNESCO. 2013; 1 (7):25-34.

7. Institución Interamericana de Derechos Humanos (IIDH). Promoción y defensa de los derechos reproductivos: nuevo reto para las instituciones nacionales de derechos humanos; IIDC, San José, 2003, 123-129.

8. Comisión Nacional de Derechos Humanos (CNDH). Recomendación General No. 31/2017. Sobre la violencia 
obstétrica en el Sistema Nacional de Salud [Internet]. México: CNDH; 31 Jul 2017 [consultado 18 Dic 2020]. Disponible en: https://www.cndh.org.mx/sites/default/files/ documentos/2019-07/RecGral_031.pdf

9. Departamento de Derecho Internacional (DEA) [Internet]. Costa Rica: DEA; 2014 [Consultado 20 Ene 2021]. Disponible en: https://www.oas.org/dil/esp/tratados_b-32_convencion_ americana_sobre_derechos_humanos.htm

10. Norma Oficial Mexicana NOM-007-SSA2-2016 [Internet]. Para la atención de la mujer durante el embarazo, el parto y el puerperio y a la persona recién nacida. Diario Oficial de la Federación. 7 abr 2016 [Consultado 8 ene 2021]. Disponible en: https://www.cndh.org.mx/sites/all/ doc/Programas/VIH/Leyes\%20y\%20normas\%20y\%20 reglamentos/Norma\%200ficial\%20Mexicana/NOM-007SSA2-2016\%20Embarazo,\%20parto\%20y\%20puerperio.pdf

11. Organización de las Naciones Unidas para la Educación, la Ciencia y la Cultura [Internet]. Reino Unido: UNESCO; 2005 (Consultado 18 Feb 2021]. Disponible en: http://portal. unesco.org/es/ev.php-URL_ID=31058\&URL_DO=DO_ TOPIC\&URL_SECTION=201.html

12. UN Women. Convention on the Elimination of All Forms of Discrimination against Women (CEDAW) [Internet]. United States: ONU; 2009 [Consulted 18 Feb 2021]. Available in: https://www.un.org/womenwatch/daw/ cedaw/cedaw.htm

13. Cámara de Diputados del H. Congreso de la Unión. Constitución Política de los Estados Unidos Mexicanos [Internet]. México; 2011 [Consultado 6 Ene 2021]. Disponible en:http://hww.diputados.gob.mx/servicios/datorele/cmprtvs/
iniciativas/Inic/36/2.htm\#: :text=El\%2Ovar\%C3\%B3n\%20y\%20 la\%20mujer,la\%20protecci\%C3\%B3n\%20de\%20la\%20salud.

14. International Mother-Baby Childbirth Organization (IMBCO) [Internet]. IMBCO and FICO created a new global Initiative. USA; Oct 2014 [Consulted 20 January 2021. Available in: http://imbco.weebly.com/

15. Norma Oficial Mexicana NOM-234-SSAT-2003 [Internet]. Utilización de campos clínicos para ciclos clínicos e internados de pregrado. Diario Oficial de la Federación. 24 ago 2003 [Consultado 8 enero 2021]. Disponible en: https://salud.qroo.gob.mx/portal/descargas/calidad/ Internado\%20M\%C3\%A9dico\%20de\%20Pregrado/ NOM-234-SSAI-2003\%20Utilizaci\%C3\%B3n\%20de\%20 campos\%20cl\%C3\%ADnicos\%20para\%20ciclos $\% 20$ cl\%C3\%ADnicos\%20e\%20internado\%20de\%20pregrado.pdf

16. Ley General de Acceso a las Mujeres a Una Vida Libre de Violencia, Gobierno de México [Internet]. México; 2015 [consultado 14 Feb 2021]. Disponible en: https://www.gob. $\mathrm{mx} /$ conavim/documentos/ley-general-de-acceso-de-lasmujeres-a-una-vida-libre-de-violencia-pdf\#: : text=enero\%20 de\%202017-,La\%20Ley\%20General\%20de\%20Acceso\%20 de\%20las\%20Mujeres\%20a\%20una,general\%20en\%20la\%20 Rep\%C3\%BAblica\%20Mexicana.\&text=Asimismo\%2C\%20 define\%20el\%20hostigamiento\%2C\%20el,cual\%20se\%20 tipifica\%20el\%20feminicidio.

17. World Health Organization (WHO). Regional Office for Europe. Essential antenatal, perinatal and postpartum care: training modules [Internet]. Copenhagen: Feb 2002 (Consulted 18 January 2021). Available in: https://apps.who. int/iris/handle/10665/107482 